\title{
Safe and tolerable one-hour pamidronate infusion for multiple myeloma patients
}

\author{
Dimitrios Chantzichristos \\ Björn Andréasson \\ Peter Johansson
}

Department of Internal Medicine, Uddevalla Hospital, Uddevalla, Sweden

Correspondence: Peter Johansson Department of Internal Medicine, Uddevalla Hospital, Fjällvägen 7 ,

45 I 80 Uddevalla, Sweden

Tel +4652292000

Fax +46 52293232

Email peter.l.johansson@vgregion.se
Background: Once a month, patients with multiple myeloma received an infusion of bisphosphonates, principally to reduce osteoclastic bone resorption. Recommended infusion time for pamidronate is 2 hours in the US and 4 hours in Europe because of its potential nephrotoxicity. From 2003, a $90 \mathrm{mg}$ infusion of pamidronate was provided over 1 hour to patients with no pre-existing renal impairment, in the Daily Care Unit at Uddevalla Hospital.

Method: Retrospective analysis of the renal deterioration, serum calcium, and adverse effects in patients with multiple myeloma treated with 1-hour pamidronate $90 \mathrm{mg}$ infusion from January 2003 to April 2007.

Results: Seventy-nine patients provided valuable data. A total number of 846 infusions were given and the median number of infusion to each patient was 11. Significant creatinine elevation was seen in 7 patients $(8.9 \%)$, after 19 infusions (2.2\%). Renal deterioration occurred in 5 of these 7 patients, which was related to progress of the myeloma or opportunistic infections. Prevalence of infusion-related events was $0.8 \%$ and the mean total S-Ca was $0.05 \mathrm{mmol} / \mathrm{L}$ lower than the baseline.

Conclusion: Few events of renal deterioration, hypocalcemia, or other adverse effects resulted from a 1-hour pamidronate $90 \mathrm{mg}$ infusion for multiple myeloma patients with no pre-existing renal impairment.

Keywords: bisphosphonates, pamidronate, multiple myeloma, infusion time

\section{Introduction}

Multiple myeloma is a B cell malignancy characterized by osteolytic bone destruction resulting from asynchronous bone turnover, that is, the increased osteoclastic resorption of bone is not accompanied by increased bone formation (Kanis et al 1991; Berenson et al 2007). Bone disease can lead to bone pain, diffuse osteopenia, focal lytic lesions, pathologic fractures, spinal cord compression, and hypercalcemia, and is a major cause of morbidity and mortality in patients with myeloma (Yeh and Berenson 2006).

Bisphosphonates, in the form of pamidronate and zoledronic acid, which have been validated as adjunctive therapy in the prevention and treatment of myeloma bone disease, inhibit osteoclastic activity and are effective in the treatment of cancer associated hypercalcemia (Berenson et al 1996). The second-generation bisphosphonate, pamidronate (pamidronate disodium), is a potent inhibitor of bone absorption at doses that do not affect bone mineralization, and is now a mainstay of myeloma therapy (Kellihan and Mangino 1992; Berenson et al 1996). The most effective dose for normalizing serum calcium concentrations in a dose-ranging trial in patients with cancer-associated hypercalcemia was found to be $90 \mathrm{mg}$ of pamidronate (Nussbaum et al 1993). For multiple myeloma patients with bone disease and no pre-existing renal impairment, newly updated guidelines (Lacy et al 2006; Kyle et al 2007) recommend an intravenous pamidronate $90 \mathrm{mg}$ infusion delivered over at least 2 hours or zoledronic acid 4 mg delivered over 50 minutes every 3 to 4 weeks for 2 years. Reducing the 
initial pamidronate dose is recommended for patients with pre-existing renal insufficiency.

Intravenous bisphosphonates are eliminated almost exclusively by the kidneys; this elimination is dependent on the patient's creatinine clearance (Mehrotra and Ruggiero 2006). Pamidronate-induced renal deterioration depends on dose, and infusion time. It usually occurs with doses over 180 mg (Markowitz et al 2001; Desikan et al 2002; Markowitz et al 2002) rather than infusion time of less than 2 hours (Leyvraz et al 1992; Janssen et al 2001; Lockridge et al 2002). In a large trial, comprising more than 1600 patients, the incidence of renal toxicity was $9.3 \%$ for pamidronate and $10.7 \%$ for zoledronic acid (Rosen et al 2001). In this clinical trial, renal deterioration occurred in $6.7 \%-10 \%$ of patients treated with a 2-hour infusion of pamidronate, depending on the renal toxicity definition.

Known pamidronate complications are fever/pyrexia, nausea, emesis, fatigue, headache, hypocalcemia, hypophosphatemia, hypomagnesemia, lymphocytopenia, thrombocytopenia, rigidity, arthralgia, and myalgia. In addition, local injection site skin reactions including pain, redness, swelling, indurations, phlebitis, and thrombophlebitis are described. Serious complications, occurring in less than $2 \%$ of patients, include acute systemic inflammatory reaction, ocular inflammation, renal failure, nephritic syndrome, electrolyte imbalance, and osteonecrosis of the maxilla and mandible (Migliorati et al 2006; Tanvetyanon and Stiff 2006).

Some recently published articles suggest that the risk of renal deterioration may not increase significantly when pamidronate is infused over 1 hour (Tyrell et al 1994). The results of the 1-hour comparator trial including 80 multiple myeloma patients conducted by de Lemos and colleagues (2007) suggest that the prevalence of renal deterioration was not significantly different in patients treated with a 2-hour infusion. Reducing pamidronate administration time would also help to reduce the average cost per treatment, and the waiting list, and benefit quality of life for the patient.

Based on early data on the safety of shorter pamidronate infusion time, patients with multiple myeloma at the outpatient clinic in Uddevalla were given 1-hour infusions from year 2003 .

\section{Method Study design}

This retrospective study, from 2003 to April 2007, included all patients treated with pamidronate, who had multiple myeloma and no pre-existing renal impairment. The study was performed in the Hematology Section of Uddevalla
Hospital, Sweden. S-creatinine (S-Cr), S-calcium (S-Ca) or $\mathrm{S}$-free ionized calcium, and $\mathrm{S}$-albumin before and during the pamidronate treatment were recorded.

\section{Assessments}

Renal deterioration during the treatment was defined as S-Cr increase $\geq 44.2 \mu \mathrm{mol} / \mathrm{L}$ or as an increase $\geq 2$ times the baseline if baseline $\mathrm{S}-\mathrm{Cr}$ was $\geq 124 \mu \mathrm{mol} / \mathrm{L}$. For those patients with baseline $\mathrm{S}-\mathrm{Cr} \geq 124 \mu \mathrm{mol} / \mathrm{L}$, renal deterioration during the treatment was defined as $\mathrm{S}-\mathrm{Cr}$ increase $\geq 88.4 \mu \mathrm{mol} / \mathrm{L}$, or as an increase $\geq 2$ times the baseline. Our laboratory has as total S-Ca reference value the range 2.32-2.62 ( $\mathrm{mmol} / \mathrm{L})$. Baseline S-Cr or S-Ca was defined as the value reported closest to the time of the first pamidronate dose within the 3 months before that dose. We assessed the increase of S-Cr and S-Ca after each treatment, and for each patient.

\section{Statistical analysis}

Standard statistical methods were employed. Mean values and standard deviation $( \pm \mathrm{SD})$ are reported. The difference between means was tested with Student's t test and the non parametric Mann-Whitney test when needed. Differences between the results in our study and comparable trials were tested with Fisher's exact test and chi square test. The difference between means was considered statistically significant if $\mathrm{p}<0.05$.

\section{Results}

We identified 79 patients who had undergone pamidronate $90 \mathrm{mg}$ therapy, and who had no pre-existing renal impairment. Their mean age was 68 years and $63 \%$ of patients were men. The mean number of pamidronate cycles per patient was 10.7. The number of infusions with evaluable data concerning $\mathrm{S}-\mathrm{Cr}$, adverse effects such as S-Ca, or S-free ionized calcium were 793, 846, and 669 respectively. The S-Cr and total S-Ca before treatment, also called the baseline, were $99.8 \mu \mathrm{mol} / \mathrm{L}$ and $2.53 \mathrm{mmol} / \mathrm{L}$ respectively. The difference in baseline $\mathrm{S}-\mathrm{Cr}$ between the sexes was significant (Table 1).

\section{Renal deterioration}

The prevalence of renal deterioration between 2 pamidronate infusions was $2.2 \%$ for the total population and $0.8 \%$ for women (not statistically significant difference). S-Cr increase at least between 2 pamidronate infusions was observed in $8.9 \%$ of the patients. The distribution of renal impairment between the sexes was similar; 5 men and 2 women (Table 2).

Analyzing the cause of creatinine increase in those 7 patients revealed that in 3 patients the increase was a 
Table I Patient demographics, number of pamidronate cycles per patient, and baseline creatinine and calcium concentrations (mean \pm SD, range within parenthesis)

\begin{tabular}{llll}
\hline & Total & Female & Male \\
\hline No of pat. identified & 79 & $29(37 \%)$ & $50(63 \%)$ \\
Mean age at diagnosis (years) & $68 \pm 10.4(38-88)$ & $70 \pm 8.7(53-86)$ & $67 \pm 1 \mathrm{I} .1(38-88)$ \\
No. of pamidronate cycles/no pat. & $10.7 \pm 7.1(\mathrm{I}-3 \mathrm{I})$ & $8.8 \pm 5.8(\mathrm{I}-25)$ & $1 \mathrm{I} .8 \pm 7.6(\mathrm{I}-3 \mathrm{I})$ \\
$\mathrm{S}-\mathrm{Cr}(\mu \mathrm{mol} / \mathrm{L})$ & $99.8 \pm 37.8(52-267)$ & $87 \pm 23.7^{\mathrm{a}}(52-160)$ & $107.5 \pm 42.7^{\mathrm{a}}(54-267)$ \\
Total S-Ca $(\mathrm{mmol} / \mathrm{L})$ & $2.53 \pm 0.19(2.24-3.02)$ & $2.5 \mathrm{I} \pm 0.14(2.24-2.94)$ & $2.54 \pm 0.21(2.24-3.02)$ \\
\hline
\end{tabular}

aThe difference in baseline $\mathrm{S}-\mathrm{Cr}$ between the two sexes is significant $(\mathrm{p}<0.03)$.

Abbreviations: $\mathrm{S}-\mathrm{Cr}$, serum creatinine; S-Ca, serum calcium.

result of progress of the disease, and that in 2 , it resulted from opportunistic infections. In the remaining 2 patients no obvious cause could be found, and the interpretation was that pamidronate-induced renal impairment was probable. These two patients received 10 and 26 infusions, respectively.

\section{Adverse effects}

The infusions of pamidronate were tolerated well. No fatal or life-threatening events occurred. Previously described adverse effects of pamidronate excluded osteonecrosis of the jaw, but included other events related to infusion (hypertension), observed in $0.8 \%$ of infusions or $7.6 \%$ of patients. One male patient was diagnosed with osteonecrosis of the jaw after less than 10 pamidronate cycles (Table 2).

\section{Hypocalcemia}

During therapy, the minimum values recorded of S-Ca and S-free ionized calcium were 2.09 and $1.13 \mathrm{mmol} / \mathrm{L}$, respectively. The mean total S-Ca was $0.05 \mathrm{mmol} / \mathrm{L}$, lower than the baseline total S-Ca (Table 2).

\section{Pamidronate therapy failure}

The incidence of this newly introduced term for pamidronate therapy, demonstrated as persistent hypercalcemia after two consecutive infusions, was $1.3 \%$. This value reduced to $0.9 \%$ when patients with progression of multiple myeloma disease were excluded.

\section{Discussion}

The 1-hour $90 \mathrm{mg}$ pamidronate infusion for patients with multiple myeloma, and no pre-existing renal impairment, was shown to be safe from the risk of nephrotoxicity and pamidronate induced hypocalcemia, and was well tolerated, in accordance with the present pamidronate therapy guidelines (Lacy et al 2006; Kyle et al 2007). Renal deterioration occurred in $8.9 \%$ of the 79 patients. The proportion of the events related to infusion, including wzzell-known infusion-related adverse effects, was approximately $10 \%$ and the incidence of therapy failure was $1.3 \%$. The comparator study conducted by de Lemos and colleagues (2007) showed that renal deterioration occurred in $15 \%$ of patients, and that the median increase in $\mathrm{S}-\mathrm{Cr}$ was $13 \%$. The difference in renal deterioration between our and the comparator study is not significant $(\mathrm{p}=0.343)$.

Table 2 Events of serum creatinine increase, infusion related events (inclusive adverse, exclusive osteonecrosis of the jaw), hypocalcemia $(<2.32 \mathrm{mmol} / \mathrm{L})$ such as $\mathrm{S}-\mathrm{Cr}$ and S-Ca range under treatment in 79 patients with multiple myeloma given one hour pamidronate $90 \mathrm{mg}$ infusion. For each parameter, the number of infusions with evaluable data are used

\begin{tabular}{llll}
\hline & Total & Female & Male \\
\hline S-Cr increasing events/no infusions & $17 / 7902.2 \%$ & $2 / 2450.8 \%$ & $15 / 5452.8 \%$ \\
S-Cr increasing events/no patients & $7 / 798.9 \%$ & $2 / 296.9 \%$ & $5 / 5010 \%$ \\
S-Cr range during treatment ( $\mu$ mol/L) & $37-333$ & $37-175$ & $44-333$ \\
Events related to the infusion/no infusions & $7 / 8460.8 \%$ & $2 / 2560.8 \%$ & $5 / 5900.9 \%$ \\
Events related to the infusion/no patients & $6 / 797.6 \%$ & $2 / 296.9 \%$ & $4 / 508 \%$ \\
Hypocalcemia events/no infusions & $69 / 66910.3 \%$ & $8 / 2093.8 \%$ & $61 / 46013.3 \%$ \\
Hypocalcemia events/no patients & $28 / 7935.4 \%$ & $6 / 2920.7 \%$ & $22 / 5044 \%$ \\
S-Ca range during treatment (mmol/L) & $2.09-3.94$ & $2.18-3.94$ & $2.09-3.28$ \\
\hline
\end{tabular}

Abbreviations: $\mathrm{S}-\mathrm{Cr}$, serum creatinine; $\mathrm{S}-\mathrm{Ca}$, serum calcium. 
Pamidronate's efficacy is also demonstrated in our trial by the low incidence of pamidronate therapy failure, defined as persistent tumor-induced hypercalcemia after two infusions in a row, independent of the progression status of multiple myeloma disease.

During the pamidronate treatment a trend towards a decrease in S-Cr was observed. No patients showed any clinical symptoms related to hypocalcemia. Comparison of the baseline mean, and mean during treatment, of S-Cr for each patient in our study population showed no significant reduction in S-Cr. However, further clinical trials are needed to fully investigate this observation. We assume that the low incidence of nephrotoxicity in our study population is due to the careful choice of patients for the decreased pamidronate infusion, based on previous studies, our clinical experience, and recommendations concerning the indications, doseoptimization, and duration of pamidronate therapy.

Because males are slightly more commonly affected by multiple myeloma, they normally represent a larger group in a study population. The other factor that contributed to fewer females in the study was our intention to treat with a lower dose individuals with low weight, based on our clinical experience. The careful selection of female individuals rather than the fewer pamidronate cycles at 8.8 compared to 11.8 (statistically insignificant difference) in men is thought to have contributed to the differences in prevalence of renal deterioration such as in the percentage of the reduction of $\mathrm{S}-\mathrm{Cr}$ between the two sexes. Renal impairment developed in $6.9 \%$ of women and $10 \%$ of men, but a reduction of more than $30 \%$ of baseline S-Cr occurred in $3.5 \%$ of women and $8 \%$ of men.

In conclusion, monthly infusions of pamidronate are an effective adjunctive treatment to palliate the destructive skeletal events often occuring in patients with multiple myeloma. One hour's treatment with $90 \mathrm{mg}$ of pamidronate in patients with no pre-existing renal impairment is proved to be safe and well tolerated, and to improve patient comfort and well-being. Our retrospective trial showed that reducing the infusion time from 4 hours to 1 hour caused no significant increase in nephrotoxicity, hypocalcemia, and adverse effects.

\section{Acknowledgment}

The study was partially economically supported by FoU-rådet Fyrbodal, Sweden and an abstract has been presented at the Swedish National Medicine Conference 2007 in Stockholm.

\section{Disclosures}

None of the authors declare conflicts of interest.

\section{References}

Berenson JR, Lichtenstein A, Porter L, et al. 1996. Efficacy of pamidronate in reducing skeletal events in patients with advanced multiple myeloma. N Engl J Med, 334:488-93.

Berenson JR, Rajdev L, Broder M. 2007. Bone complications in multiple myeloma. Cancer Biol Ther, 5:1082-5.

de Lemos ML, Taylor SC, Barnett JB, et al. 2007. Renal safety of one hour pamidronate infusion for breast cancer and multiple myeloma patients: comparison between clinical trials and population-based database. J Oncol Pharm Prac, 12:193-9.

Desikan R, Veksler Y, Raza S, et al. 2002. Nephrotic proteinuria associated with high-dose pamidronate in multiple myeloma. $\mathrm{Br} J$ Haematol, 119: 496-9.

Janssen van Doorn K, Neyns B, Van der Niepen P, et al. 2001. Pamidronate-related nephrotoxicity (tubulointerstitial nephritis) in a patient with osteolytic bone metastases. Nephron, 89:467-8 .

Kanis JA, McCloskey EV, Taube T, et al. 1991. Rationale for the use of bisphosphonates in bone metastases. Bone, 12:13-8.

Kellihan MJ, Mangino PD. 1992 Pamidronate. Ann Pharmacother, 26:1262-9.

Kyle RA, Yee GC, Somerfield MR, et al. 2007. American Society of Clinical Oncology 2007 clinical practice guideline update on the role of bisphosphonates in multiple myeloma. J Clin Oncol, 25:2464-72.

Lacy MQ, Dispenzieri A, Gertz MA, et al. 2006. Mayo clinic consensus statement for the use of bisphosphonates in multiple myeloma. Mayo Clin Proc, 81:1047-53.

Leyvraz S, Hess U, Flesch G, et al. 1992. Pharmacokinetics of pamidronate in patients with bone metastases. $J$ Nat Cancer Inst, 84:788-92.

Lockridge L, Papac RJ, Perazella MA. 2002. Pamidronate-associated nephrotoxicity in a patient with Lagerhans's histiocytosis. Am J Kidney Dis, 40:E2.

Markowitz GS, Appel GB, Fine PL, et al. 2001. Collapsing focal segmental glomerulosclerosis following treatment with high-dose pamidronate. J Am Soc Nephrol, 12:1164-72.

Markowitz GS, Fine PL, D'Agati VD. 2002. Nephrotic syndrome after treatment with pamidronate. Am J Kidney Dis, 39:1118-22.

Mehrotra B, Ruggiero S. 2006. Bisphosphonate complications including osteonecrosis of the jaw. Hematology, American Society of Hematology, Education Program Book, 356-60.

Migliorati CA, Siegel MA, Elting LS. 2006. Bisphosphonate-associated osteonecrosis: a long-term complication of bisphosphonate treatment. Lancet Oncol, 7:508-14.

Nussbaum Sr, Younger J, Vandepol Cj, et al. 1993. Single-dose intravenous therapy with pamidronate for the treatment of hypercalcemia of malignancy: comparison of 30-, 60-, and 90-mg dosages. Am J Med, 95:297-304

Rosen LS, Gordon D, Kaminski M, et al. 2001. Zoledronic acid versus pamidronate in the treatment of skeletal metastases in patients with breast cancer or osteolytic lesions of multiple myeloma: a phase III, double-blind, comparative trial. Cancer J, 7:377-87.

Tanvetyanon T, Stiff PJ. 2006. Management of the adverse effects associated with intravenous bisphosphonates. Ann Oncol, 17:6:897-907.

Tyrell CJ Collinson M, Madsen EL, et al. 1994. Intravenous pamidronate: infusion rate and safety. Ann Oncol, 5:27-29.

Yeh HS, Berenson JR. 2006. Treatment for myeloma bone disease. Clinical Cancer Research. 12:6279-84. 